\title{
Systemic Delivery of Small Interfering RNA by Use of Targeted Polycation Liposomes for Cancer Therapy
}

\author{
Eriya Kenjo, ${ }^{a}$ Tomohiro Asai, ${ }^{a}$ Norihito Yonenaga ${ }^{a}$ Hidenori Ando, ${ }^{a}$ Takayuki Ishii, ${ }^{a}$ \\ Kentaro Hatanaka, ${ }^{a}$ Kosuke Shimizu, ${ }^{a}$ Yugo Urita, ${ }^{b}$ Takehisa Dewa, ${ }^{b}$ Mamoru Nango, ${ }^{b}$ \\ Hideo Tsukada, ${ }^{c}$ and Naoto $\mathrm{Oku}^{*, a}$ \\ ${ }^{a}$ Department of Medical Biochemistry, School of Pharmaceutical Sciences, University of Shizuoka; 52-1 Yada, \\ Suruga-ku, Shizuoka 422-8526, Japan: ${ }^{b}$ Department of Frontier Materials, Graduate School of Engineering, Nagoya \\ Institute of Technology; Gokiso-cho, Showa-ku, Nagoya, Aichi 466-8555, Japan: and ${ }^{c}$ PET Center, Central Research \\ Laboratory, Hamamatsu Photonics K.K.; Hirakuchi, Hamakita-ku, Hamamatsu, Shizuoka 434-8601, Japan. \\ Received September 18, 2012; accepted November 27, 2012
}

Novel polycation liposomes decorated with cyclic(Cys-Arg-Gly-Asp-D-Phe) peptide (cyclicRGD)-polyethylene glycol (PEG) (RGD-PEG-polycation liposomes (PCL)) were previously developed for cancer therapy based on RNA interference. Here, we demonstrate the in vivo delivery of small interfering RNA (siRNA) to tumors by use of RGD-PEG-PCL in B16F10 melanoma-bearing mice. Pharmacokinetic data obtained by positron emission tomography showed that cholesterol-conjugated siRNA formulated in RGD-PEG-PCL markedly accumulated in the tumors. Delivered by RGD-PEG-PCL, a therapeutic cocktail of siRNAs composed of cholesterol-conjugated siRNAs for c-myc, MDM2, and vascular endothelial growth factor (VEGF) were able to significantly inhibit the growth of B16F10 melanoma both in vitro and in vivo. These data suggest that targeted delivery of siRNAs by use of RGD-PEG-PCL has considerable potential for cancer treatment.

Key words small interfering RNA; liposome; polycation; cancer therapy; positron emission tomography

RNA interference (RNAi), which is sequence-dependent post-transcriptional gene silencing, is caused by doublestranded RNA and plays an important role in the regulation of protein expression. ${ }^{1)}$ Synthetic small interfering RNA (siRNA) has been widely used for gene silencing in scientific research and is expected to become a beneficial drug for various diseases. $^{2-4)}$ Although siRNA shows efficient gene silencing, the use of it is quite limited in vivo, especially for systemic usage, because of the rapid degradation of RNA in the bloodstream by nucleases, difficulty in delivery of it to target tissues, and its poor entry into the cytosol of target cells. ${ }^{4)}$ Therefore, establishment of a systemic siRNA delivery system that enables prolonged circulation without degradation by nucleases, active targeted delivery to the target tissue, and efficient entry of the siRNA into the target cells has been awaited.

Polycation liposomes (PCL) possessing useful properties of both cationic liposomes and polycations have been developed for delivery of nucleic acids such as plasmid DNA and siRNA. ${ }^{5-8)}$ Among various PCL composed of different kinds of polycations, PCL containing dicetylphosphate-tetraethylenepentamine (DCP-TEPA) as a main component have been found to be quite efficient for siRNA delivery and for gene silencing. ${ }^{8)}$ DCP-TEPA-based PCL (TEPA-PCL) modified with polyethylene glycol (PEG) show the property of long circulation in vivo after intravenous (i.v.) injection. Although PEGylation of siRNA/TEPA-PCL complexes causes partial dissociation of the siRNA from the complex during the PEGylation step, this dissociation was found to be avoidable by conjugating cholesterol to the 3 '-end of the sense strand of the siRNA (siRNA-C). ${ }^{8)}$ For active targeted delivery of siRNA to tumor tissues, we earlier investigated the usefulness of modification of these PEGylated TEPA-PCL (PEG-PCL) with cyclic(Cys-Arg-Gly-Asp-D-Phe) peptide (cyclicRGD) and termed them RGD-PEG-PCL. ${ }^{9)}$ The RGD motif is known to

The authors declare no conflict of interest. bind $\alpha \mathrm{v}$ integrins expressed on tumor and angiogenic endothelial cells. ${ }^{10,11)}$ By use of mice bearing B16F10-luc2 murine melanoma stably expressing the luciferase 2 gene (Luc2), we observed the knockdown of luciferase expression in vivo after systemic injection of cholesterol-conjugated siRNA for Luc2 (siLuc2-C) carried by RGD-PEG-PCL.)

In the present study, we used these RGD-PEG-PCL for delivering therapeutic siRNA for cancer treatment. Song et al. previously reported that siRNAs for c-myc, MDM2, and vascular endothelial growth factor (VEGF) suppressed B16 tumor growth $^{12)}$; and Li et al. also observed inhibition of B16F10 metastasis with this siRNA cocktail. ${ }^{13)}$ Therefore, we used this therapeutic siRNA cocktail for the treatment of tumors to evaluate the usefulness of RGD-PEG-PCL as an siRNA carrier for systemic and active targeted delivery.

\section{MATERIALS AND METHODS}

Materials The synthetic polycationic lipid DCP-TEPA was synthesized as described previously. ${ }^{8)}$ Briefly, dimerized dicetylphosphate anhydride in anhydrous chloroform was added to a solution of tetraethylenepentamine (SigmaAldrich, St. Louis, MO, U.S.A.) in anhydrous pyridine, and the reaction mixture was stirred for $4 \mathrm{~h}$. After the removal of the solvent by evaporation, the residue was resuspended in distilled water and filtered to remove any unreacted tetraethylenepentamine. DCP-TEPA was purified by column chromatography using aminated silica gel (Chromatorex $\mathrm{NH}$, Fuji Silisia Chemical Ltd., Aichi, Japan). Dipalmitoylphosphatidylcholine (DPPC) and cholesterol were kindly provided by Nippon Fine Chemical Co., Ltd. (Takasago, Hyogo, Japan). Dioleoylphosphatidylethanolamine (DOPE), distearoylphosphatidylethanolamine-polyethyleneglycol5000 (DSPE-PEG), and DSPE-PEG5000-maleimide were obtained from NOF Co., Ltd. (Tokyo, Japan). CyclicRGD peptide was purchased from Operon Biotechnologies (Tokyo, Japan) and conjugated to 
DSPE-PEG5000-maleimide (1:1 as a molar ratio) by incubation for $30 \mathrm{~min}$ at room temperature.

Cholesterol-conjugated siRNAs (siRNA-C) were purchased from Hokkaido System Science Co., Ltd. (Hokkaido, Japan), where cholesterol was conjugated to the siRNA at the 3'end of its sense strand. The nucleotide sequences of siRNA with a 2-nucleotide overhang (underlined) were the following: 5'-GCUAUGGGCUGAAUACAAAdTdT-3' (sense) and 5'-UUUGUAUUCAGCCCAUAGCdTdT-3' (antisense) for luciferase 2 (siLuc2); 5'-GAACAUCAUCAUCCAGGACdTdT-3' (sense) and 5'-GUCCUGGAUGAUGAUGUUCdTdT-3' (antisense) for c-myc; 5'-CGAUGAAGCCCUGGAGUGCdTdT-3' (sense) and 5'-GCUACUUCGGGACCUCACGdTdT-3' (antisense) for VEGF; 5'-GCUUCGG AAC AAG AGACUCdTdT-3' (sense) and 5'-UCGAAGUACUCAGCGUAAGdTdT-3' (antisense) for MDM2; and a scrambled sequence (siCont), 5'CGAUUCGCUAGACCGGCUUCAUUGCAG-3' (sense) and 5'GCAAUGAAGCCGGUCUAGCGAAUCGAU-3' (antisense), for the control. For the pharmacokinetic study using positron emission tomography, $\left[{ }^{18} \mathrm{~F}\right]$-labeled siRNA conjugated with cholesterol ([ $\left.{ }^{18} \mathrm{~F}\right]$ siRNA-C) was prepared as described previously. ${ }^{14)}$

Preparation of PEGylated or RGD-PEGylated TEPAPCL Bearing siRNAs siRNA-C formulated in PEGPCL or RGD-PEG-PCL was prepared as described previously. ${ }^{9)}$ In brief, DCP-TEPA, DOPE, DPPC, and cholesterol $(0.25: 1: 0.75: 1)$ were dissolved in $t$-butyl alcohol for freezedrying, and hydrated in RNase-free water. The prepared TEPA-PCL were extruded 10 times through a polycarbonate membrane filter with 100-nm pores (Nuclepore, Maidstone, U.K.) by using an extruder (Lipex, Vancouver, Canada). The TEPA-PCL were then incubated with siRNA-C for $20 \mathrm{~min}$ to form siRNA-C/TEPA-PCL complexes. The ratio of the nitrogen moiety of TEPA-PCL to the phosphorus one of siRNA-C (N/P ratio) was fixed at 18 eq. Then, the siRNA-C/ TEPA-PCL complexes were PEGylated $(10 \mathrm{~mol} \%$ of the total lipids) by incubation with DSPE-PEG (siRNA-C/PEG-PCL) or with cyclicRGD-grafted DSPE-PEG (siRNA-C/RGD-PEG$\mathrm{PCL}$ ) in RNase-free water at $37^{\circ} \mathrm{C}$ for $20 \mathrm{~min}$. The particle size and $\zeta$-potential of siRNA-C/PCL were determined with a Zeta Sizer Nano ZS (Malvern, Worcs, U.K.), and found to be $129 \mathrm{~nm}$ and $-1.1 \mathrm{mV}$, respectively, for siRNA-C/PEG-PCL and $133 \mathrm{~nm}$ and $-3.1 \mathrm{mV}$, respectively, for siRNA-C/RGD-PEGPCL.

Cell Culture B16F10 murine melanoma cells were obtained from the American Type Culture Collection; and B16F10-luc2 Bioware Ultra Cell Line, a luciferase-expressing cell line stably transfected with the firefly luciferase gene (Luc2), were purchased from Caliper Life Sciences (Hopkinton, MA, U.S.A.). These cells were cultured in DME/Ham F12 medium (WAKO, Osaka, Japan) supplemented with 10\% fetal bovine serum (FBS, Sigma-Aldrich), 100 units $/ \mathrm{mL}$ penicillin (MP Biomedicals, Irvine, CA, U.S.A.), and $100 \mu \mathrm{g} / \mathrm{mL}$ streptomycin (MP Biomedicals) in a $\mathrm{CO}_{2}$ incubator.

Antiproliferation Assay in Vitro B16F10 cells $\left(4 \times 10^{4}\right.$ cells $/ 0.5 \mathrm{~mL} /$ well) were seeded into a 24 -well plate (BD Bioscience, San Jose, CA, U.S.A.) and precultured overnight. After the medium had been changed to fresh medium containing $10 \%$ FBS without antibiotics, the cells were incubated for 24,48 or $72 \mathrm{~h}$ at $37^{\circ} \mathrm{C}$ with $25 \mathrm{pmol}$ siCocktail-C (siRNAs for c-myc, MDM2, and VEGF were mixed at a $1: 1: 1$ ratio) or with 25 pmol siCont-C formulated in PEG-PCL or RGDPEG-PCL at a final siRNA concentration of $50 \mathrm{~nm}$. Cell viability was measured by performing a modified 3-(4,5-dimethylthiazol-2-yl)-2,5-diphenyltetrazolium bromide (MTT) assay using Tetracolor ONE ${ }^{\mathrm{TM}}$ (Seikagaku Co., Tokyo, Japan) in accordance with the manufacturer's instructions. The amount of formazan formed in $30 \mathrm{~min}$ was measured with a Tecan Infinite M200 micro plate reader (Salzburg, Austria) at a test wavelength of $450 \mathrm{~nm}$ and a reference wavelength of $630 \mathrm{~nm}$.

Pharmacokinetic Analysis of $\left[{ }^{18}\right.$ F]siRNA-C Determined by Positron Emission Tomography (PET) C57BL/6 male mice were obtained from Japan SLC Inc. (Shizuoka, Japan). The animals were cared for according to the Animal Facility Guidelines of the University of Shizuoka. All animal experiments were approved by the Animal and Ethics Committee of the University of Shizuoka. B16F10 cells $\left(1 \times 10^{5}\right.$ cells/mouse $)$ were injected via a tail vein into 6-week-old C57BL/6 male mice. At day 21 after the tumor cell inoculation, the mice were intravenously injected with free $\left[{ }^{18} \mathrm{~F}\right]$ siCont-C, $\left[{ }^{18} \mathrm{~F}\right]-$ siCont-C/PEG-PCL or $\left[{ }^{18} \mathrm{~F}\right]$ siCont-C/RGD-PEG-PCL (5 MBq/ mouse) under pentobarbital anesthesia. Then, the PET scan was started immediately after the injection and continued for $60 \mathrm{~min}$ by using a Clairvivo PET apparatus (Shimadzu, Kyoto, Japan).

Therapeutic Experiment Six-week-old C57BL/6 male mice were injected with B16F10-luc2 cells $\left(1 \times 10^{5}\right.$ cells/ mouse) via a tail vein. At day 12, 15, and 18 after the tumor cell inoculation, the mice were given an intravenous injection of phosphate-buffered saline (PBS) (control), siCont-C/ RGD-PEG-PCL, siCocktail-C/PEG-PCL, or siCocktail-C/ RGD-PEG-PCL ( $2 \mathrm{mg} / \mathrm{kg}$ mouse as siRNA-C). For the assessment of tumor growth by the expression of luciferase, $3 \mathrm{mg}$ luciferin (Promega KK, Tokyo, Japan) in $0.2 \mathrm{~mL}$ PBS was injected intraperitoneally (i.p.) at day 12, 15, 18, and 21 after the injection of B16F10-luc2 cells; and the luminescence intensity from the mice was measured $15 \mathrm{~min}$ post luciferin injection with a Xenogen IVIS Lumina System coupled with Living Image software for data acquisition (Xenogen, Co., Alameda, CA, U.S.A.). At day 21, all animals were sacrificed under deep anesthesia; and their lungs were excised and weighed. Lungs from normal mice of the same age were also weighed as a control.

Statistical Analysis Differences between groups were evaluated by analysis of variance (ANOVA) with the Tukey post-hoc test.

\section{RESULTS}

Antiproliferative Effect of siRNA-C Formulated in RGD-PEG-PCL on B16F10 Cells We previously reported that fluorescein isothiocyanate (FITC)-labeled cholesterolconjugated siRNA for luciferase (siLuc2-C) was effectively associated with B16F10 cells by complexing it with RGD-PEGPCL and that high efficacy of gene silencing of siLuc2-C was observed in B16F10-Luc2 murine melanoma cells by using this formulation. ${ }^{9)}$ Here, we investigated the antiproliferative effect of the therapeutic siCocktail-C targeting c-myc, MDM2, and VEGF carried by this formulation. As shown in Fig. 1, siCocktail-C/RGD-PEG-PCL significantly suppressed the proliferation of B16F10 cells in comparison with siCont-C/ RGD-PEG-PCL at $48 \mathrm{~h}$ after transfection, and in comparison 


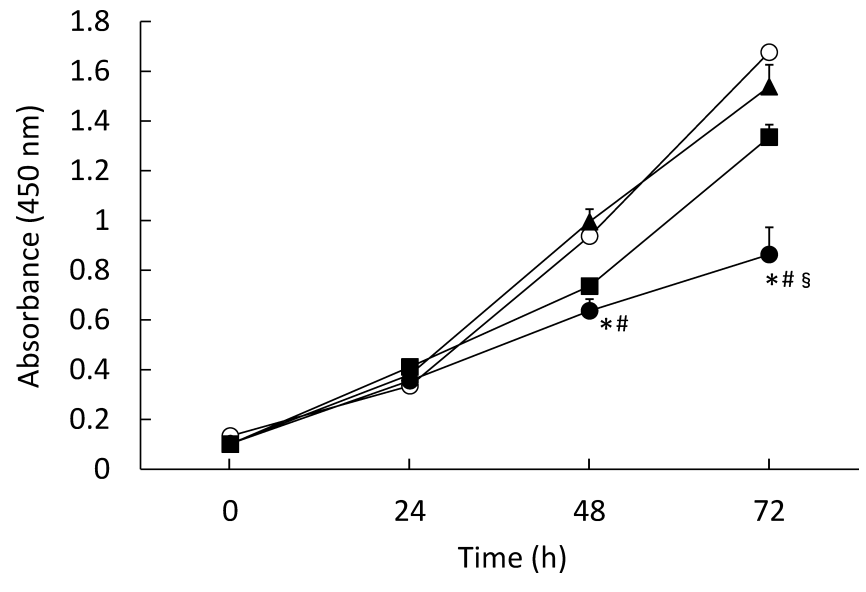

Fig. 1. Antiproliferative Effect of siCocktail/RGD-PEG-PCL on B16F10 Cells

TEPA-PCL were complexed with the mixture of cholesterol-conjugated siRNAs for c-myc, MDM2, and VEGF (1:1:1, total $25 \mathrm{pmol})$ or with siCont-C (25 pmol), and then was modified with DSPE-PEG or DSPE-PEG-RGD. B16F10 cells were incubated for $24 \mathrm{~h}$ with RNase-free water (o), siCont-C/RGD-PEG-PCL ( $(\boldsymbol{\Delta})$,

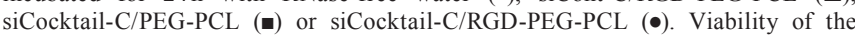
cells was estimated at the times indicated in the figure by conducting a modified assay using Tetracolor ONETM $(n=4)$. * Indicates $p<0.01 v s$. control; ${ }^{\#} p<0.01 v s$. siCont-C/RGD-PEG-PCL; and ${ }^{\S} p<0.01$ vs. siCocktail-C/PEG-PCL.

with it and siCocktail-C/PEG-PCL at $72 \mathrm{~h}$. Cell growth was quite similar between control and siCont-C/RGD-PEG-PCLtreated cells, indicating that the antiproliferative activity of the siCocktail-C/RGD-PEG-PCL was not due to cytotoxic or off-target effects.

PET Study of siRNA-C/RGD-PEG-PCL in B16F10Bearing Mice Pharmacokinetics of $\left[{ }^{18} \mathrm{~F}\right] \mathrm{siRNA}-\mathrm{C}$ formu- lated in PEG-PCL or RGD-PEG-PCL was determined by use of PET. The labeling method was previously developed by us, which enables direct labeling of double-stranded RNA. ${ }^{14)}$ $\left[{ }^{18} \mathrm{~F}\right]$ siRNA-C formulated in RGD-PEG-PCL highly accumulated in the lungs bearing metastatic colonies of B16F10 melanoma, in comparison to free $\left[{ }^{18} \mathrm{~F}\right] \mathrm{siRNA}-\mathrm{C}$ and that formulated in PEG-PCL (Fig. 2). Free $\left[{ }^{18} \mathrm{~F}\right] \mathrm{siRNA}-\mathrm{C}$ was eliminated from the body faster than $\left[{ }^{18} \mathrm{~F}\right] \mathrm{siRNA}-\mathrm{C} / \mathrm{PEG}-\mathrm{PCL}$ and $\left.{ }^{18} \mathrm{~F}\right]$ siRNA-C/RGD-PEG-PCL and accumulated less in the tumors. PEGylation of PCL increased the half-life of complexed $\left[{ }^{18} \mathrm{~F}\right]$ siRNA-C (data not shown), and the accumulation of $\left[{ }^{18} \mathrm{~F}\right]$ siRNA-C in the tumors was greater with RGD-PEGPCL than with PEG-PCL. The accumulation of $\left[{ }^{18} \mathrm{~F}\right]$ siRNA-C in other organs such as heart, liver, spleen, and kidney was similar between PEG-PCL and RGD-PEG-PCL groups (data not shown). Actual radioactivities of ${ }^{18} \mathrm{~F}$ in each organ invasively determined after PET analysis were consistent with the corresponding image data.

Therapeutic Effect of siCocktail-C/RGD-PEG-PCL on B16F10-luc2-Bearing Mice Along with the growth of the metastatic cancer, the luminescence intensity of the control mice increased time-dependently under the experimental schedule used (Fig. 3A). An increase in luminescence intensity was also observed in mice treated with siCont-C/RGDPEG-PCL. In contrast, siCocktail-C/PEG-PCL- and siCocktail-C/RGD-PEG-PCL-treated groups showed a suppressed increase in luminescence intensity. Corresponding to this, the lung colonization of B16F10 cells was suppressed in siCocktail-C/PEG-PCL- and siCocktail-C/RGD-PEG-PCL-treated groups (Fig. 3B). Quantitative data on weight gain in the lungs bearing metastatic colonies showed that siCocktail-C/

$\left[{ }^{18} \mathrm{~F}\right] \operatorname{siRNA-C}$
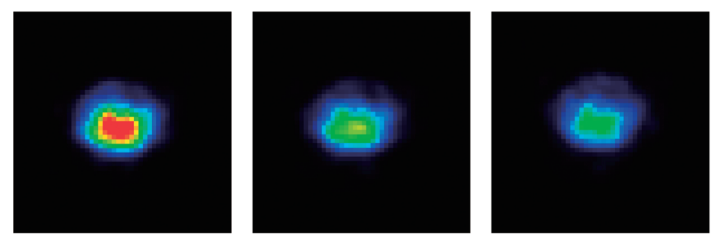

$\left[{ }^{18} \mathrm{~F}\right] \mathrm{siRNA}-\mathrm{C} / \mathrm{PEG}-\mathrm{PCL}$
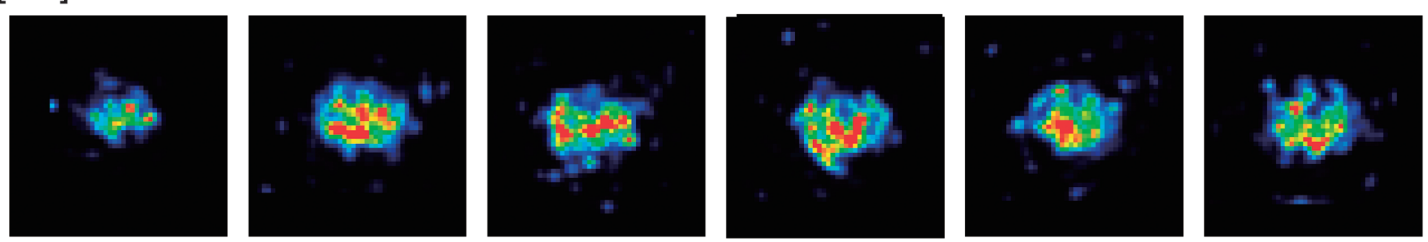

$\left[{ }^{18} \mathrm{~F}\right] \mathrm{siRNA}-\mathrm{C} / \mathrm{RGD}-\mathrm{PEG}-\mathrm{PCL}$

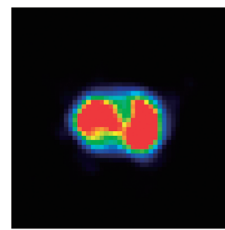

$1-5$

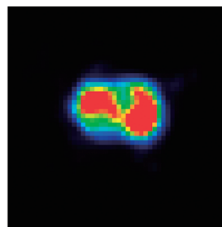

$11-15$

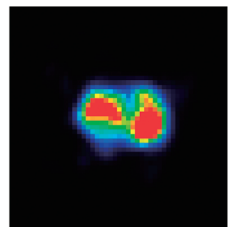

$21-25$

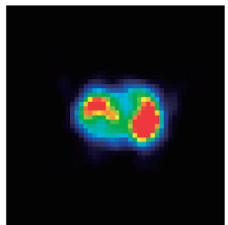

$31-35$

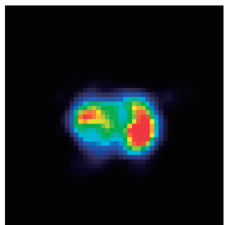

$41-45$

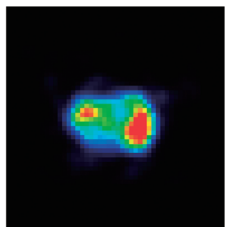

$51-55(\min )$

Fig. 2. PET Imaging of $\left[{ }^{18} \mathrm{~F}\right]$ siRNA Distribution in the Lungs Bearing B16F10 Melanoma

$\left[{ }^{18} \mathrm{~F}\right] \mathrm{siRNA}-\mathrm{C},\left[{ }^{18} \mathrm{~F}\right] \mathrm{siRNA}-\mathrm{C} / \mathrm{PEG}-\mathrm{PCL}$, or $\left[{ }^{18} \mathrm{~F}\right] \mathrm{siRNA}-\mathrm{C} / \mathrm{RGD}-\mathrm{PEG}-\mathrm{PCL}(5 \mathrm{MBq}$ in $0.2 \mathrm{~mL})$ were intravenously administered via a tail vein to C57BL/6 male mice bearing B16F10 metastatic tumors. PET images were acquired with cross-section frames for $60 \mathrm{~min}$ after the administration of [ $\left.{ }^{18} \mathrm{~F}\right] \mathrm{siRNA}$ samples. The images of the lungs bearing metastatic tumors are shown. PET data were obtained from 3 independent animal experiments, all of which showed similar results. 

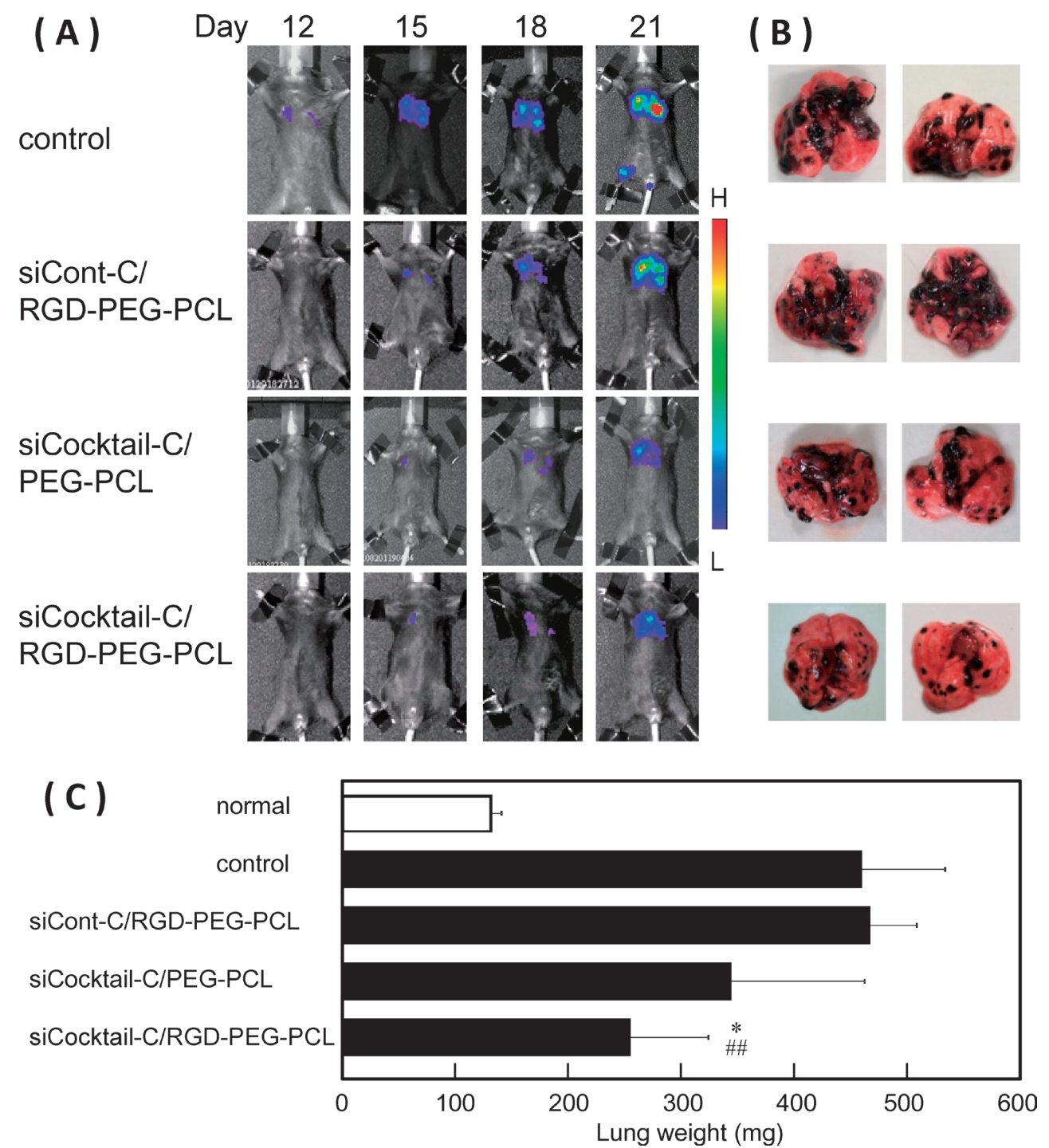

Fig. 3. Therapeutic Effect of siCocktail/RGD-PEG-PCL in the B16F10-luc2 Metastatic Tumor-Bearing Mice

TEPA-PCL was complexed with the mixture of cholesterol-conjugated siRNAs for c-myc, MDM2, and VEGF $(1: 1: 1)$, and then was modified with DSPE-PEG or DSPEPEG-RGD. These complexes ( $2 \mathrm{mg} / \mathrm{kg}$ as siRNA-C) were administered intravenously to C57BL/6 mice at 12,15 , and $18 \mathrm{~d}$ after the inoculation of B16F10-luc2 cells into the mice. (A) Luciferase activity in the tumor-metastasized lungs was measured by IVIS on the indicated days. The color bar at the right represents signal intensity code over the body surface area. (B) Appearance of lungs excised from the tumor-bearing mice $(n=4)$ on day 21 after tumor injection. Typical photos are shown, and the appearance of the lungs from all other mice in the same groups was similar. (C) Lung weight on day 21 post tumor injection. *Indicates $p<0.05 v s$. control; \# indicates $p<0.01 v s$. siCont-C/RGD-PEG-PCL. Similar results were obtained in a separate experiment.

RGD-PEG-PCL significantly suppressed the growth of the metastatic tumors (Fig. 3C). The percent growth inhibition of siCocktail-C/PEG-PCL and siCocktail-C/RGD-PEG-PCL was $35.3 \%$ and $62.6 \%$, respectively.

\section{DISCUSSION}

RNAi has potential for the treatment of various diseases, since it can specifically suppress protein expression. However, systemic injection of siRNA is quite limited; since naked siRNA is readily degraded by nucleases in the bloodstream. In addition, the entry of siRNA into the cytosol of target cells requires an appropriate delivery system. We previously developed TEPA-PCL, liposomes containing DCP-conjugated synthetic polycation, for the purpose of systemic administration of siRNA. ${ }^{8)}$ In the present study, we used siRNA with cholesterol conjugated to it at the 3 '-end of its sense strand, which modification is known to have no effect on the knockdown of specific protein expression, ${ }^{9}$ for the stabilization of siRNA complexed with carrier PCL in the bloodstream. Thus, siRNA-C is considered to be internalized by cells as a complexed form.

For the active targeted delivery of siRNA, cyclic(Cys-Arg-Gly-Asp-D-Phe), originally reported by Pfaff et al., ${ }^{15}$ ) was used to decorate the surface of PEG-PCL; as this RGD motif, as mentioned earlier, is known to interact with $\alpha \mathrm{v}$ integrins highly expressed on cancer and angiogenic endothelial cells. As we previously reported, ${ }^{9)}$ RGD modification contributes to the association between our siRNA vector and target cancer cells, resulting in enhanced internalization of siRNA into these cells. Our present data from the biodistribution study using $\left[{ }^{18} \mathrm{~F}\right]$ siRNA-C showed higher accumulation of $\left[{ }^{18} \mathrm{~F}\right] \mathrm{siRNA}-\mathrm{C}$ in the tumor with RGD-PEG-PCL than with PEG-PCL. In addition, our previous study indicated that siLuc2-C formulated in RGD-PEG-PCL effectively silenced luciferase expression in lung-metastasized B16F10-luc2 
melanoma in vivo. ${ }^{9)}$ In the case of $\left[{ }^{18} \mathrm{~F}\right] \mathrm{siRNA}-\mathrm{C} / \mathrm{PEG}-\mathrm{PCL}$, the PET images did not clearly overlap lung shape. We do not know the reason for this observation at present; however, it is possible that the circulating $\left[{ }^{18} \mathrm{~F}\right]$ siRNA-C/PEG-PCL in the bloodstream caused unclear images. In contrast, the clear images of $\left[{ }^{18} \mathrm{~F}\right]$ siRNA-C/RGD-PEG-PCL distribution were obtained possibly due to ${ }^{18} \mathrm{~F}$ associated with tumor and angiogenic endothelial cells.

In the present study, we demonstrated that siCocktail-C/ RGD-PEG-PCL suppressed metastatic tumor growth after i.v. injection. Gene-silencing effects of the siCocktail for myc, MDM-2, and VEGF have already been demonstrated. ${ }^{12,13)}$ Since siCont-C/RGD-PEG-PCL did not show any antitumor activity at all, the effect of siCocktail-C/RGD-PEG-PCL on the tumor cannot be attributed to toxicity of the complex. Thus, we conclude RGD-PEG-PCL to be useful for the treatment of cancer with siRNA that suppresses the expression of target protein(s) crucial for tumor growth. Our delivery system is applicable to a number of therapeutic siRNA candidates that have been studied for cancer treatment. ${ }^{16-18)}$ The next step is to select an appropriate siRNA among these candidates and use it with the present formulation to test its suitability for use as a cancer treatment.

Acknowledgement This research was supported by a Grant-in-Aid for Scientific Research from Japan Society for the Promotion of Science.

\section{REFERENCES}

1) Hutvágner G, Zamore PD. A microRNA in a multiple-turnover RNAi enzyme complex. Science, 297, 2056-2060 (2002).

2) Davis ME, Zuckerman JE, Choi CH, Seligson D, Tolcher A, Alabi CA, Yen Y, Heidel JD, Ribas A. Evidence of RNAi in humans from systemically administered siRNA via targeted nanoparticles. $\mathrm{Na}$ ture, 464, 1067-1070 (2010).

3) Chen SH, Zhaori G. Potential clinical applications of siRNA technique: benefits and limitations. Eur. J. Clin. Invest., 41, 221-232 (2011).

4) Pecot CV, Calin GA, Coleman RL, Lopez-Berestein G, Sood AK. RNA interference in the clinic: challenges and future directions. Nat. Rev. Cancer, 11, 59-67 (2011).

5) Yamazaki $Y$, Nango M, Matsuura M, Hasegawa $Y$, Hasegawa M, Oku N. Polycation liposomes, a novel nonviral gene transfer system, constructed from cetylated polyethylenimine. Gene Ther., 7, 1148-1155 (2000).
6) Oku N, Yamazaki Y, Matsuura M, Sugiyama M, Hasegawa M, Nango M. A novel non-viral gene transfer system, polycation liposomes. Adv. Drug Deliv. Rev., 52, 209-218 (2001).

7) Asai T, Suzuki Y, Matsushita S, Yonezawa S, Yokota J, Katanasaka Y, Ishida T, Dewa T, Kiwada H, Nango M, Oku N. Disappearance of the angiogenic potential of endothelial cells caused by Argonaute 2 knockdown. Biochem. Biophys. Res. Commun., 368, 243-248 (2008).

8) Asai T, Matsushita S, Kenjo E, Tsuzuku T, Yonenaga N, Koide H, Hatanaka K, Dewa T, Nango M, Maeda N, Kikuchi H, Oku N. Dicetyl phosphate-tetraethylenepentamine-based liposomes for systemic siRNA delivery. Bioconjug. Chem., 22, 429-435 (2011).

9) Yonenaga N, Kenjo E, Asai T, Tsuruta A, Shimizu K, Dewa T, Nango M, Oku N. RGD-based active targeting of novel polycation liposomes bearing siRNA for cancer treatment. J. Control. Release, 160, 177-181 (2012).

10) Ruoslahti E. RGD and other recognition sequences for integrins. Annu. Rev. Cell Dev. Biol., 12, 697-715 (1996).

11) Arap W, Pasqualini R, Ruoslahti E. Cancer treatment by targeted drug delivery to tumor vasculature in a mouse model. Science, $\mathbf{2 7 9}$, 377-380 (1998).

12) Song E, Zhu P, Lee SK, Chowdhury D, Kussman S, Dykxhoorn DM, Feng Y, Palliser D, Weiner DB, Shankar P, Marasco WA, Lieberman J. Antibody mediated in vivo delivery of small interfering RNAs via cell-surface receptors. Nat. Biotechnol., 23, 709-717 (2005).

13) Li SD, Chono S, Huang L. Efficient oncogene silencing and metastasis inhibition via systemic delivery of siRNA. Mol. Ther., 16, 942-946 (2008).

14) Hatanaka K, Asai T, Koide H, Kenjo E, Tsuzuku T, Harada N, Tsukada H, Oku N. Development of double-stranded siRNA labeling method using positron emitter and its in vivo trafficking analyzed by positron emission tomography. Bioconjug. Chem., 21, 756-763 (2010).

15) Pfaff M, Tangemann K, Müller B, Gurrath M, Müller G, Kessler H, Timpl R, Engel J. Selective recognition of cyclic RGD peptides of NMR defined conformation by alpha IIb beta 3, alpha V beta 3, and alpha 5 beta 1 integrins. J. Biol. Chem., 269, 20233-20238 (1994).

16) Petrocca F, Lieberman J. Promise and challenge of RNA interference-based therapy for cancer. J. Clin. Oncol., 29, 747-754 (2011).

17) Wang Z, Rao DD, Senzer N, Nemunaitis J. RNA interference and cancer therapy. Pharm. Res., 28, 2983-2995 (2011).

18) Koide H, Asai T, Furuya K, Tsuzuku T, Kato H, Dewa T, Nango M, Maeda N, Oku N. Inhibition of Akt (ser473) phosphorylation and rapamycin-resistant cell growth by knockdown of mammalian target of rapamycin with small interfering RNA in vascular endothelial growth factor receptor-1-targeting vector. Biol. Pharm. Bull., 34, 602-608 (2011). 